\title{
Time-course changes of catabolic proteins following muscle atrophy induced by dexamethasone
}

\author{
Anderson G. Macedo ${ }^{a}$, André Luis O. Krug a , Lidiane M. Souza ${ }^{\mathrm{b}}$, Aline M. Martuscelli ${ }^{\mathrm{a}}$, \\ Paula B. Constantino ${ }^{a}$, Anderson S. Zago ${ }^{b}$, James W.E. Rush ${ }^{c}$, Carlos F. Santos ${ }^{\mathrm{d}}$, Sandra L. Amaral ${ }^{\mathrm{a}, \mathrm{b}, *}$ \\ a Joint Graduate Program in Physiological Sciences, PIPGCF UFSCar/UNESP, Department of Physiological Sciences, Federal University of São Carlos - UFSCAR, São Carlos, Brazil \\ ${ }^{\mathrm{b}}$ Department of Physical Education, Universidade Estadual Paulista - UNESP, Bauru, Brazil \\ ${ }^{\mathrm{c}}$ Department of Kinesiology, Faculty of Applied Health Sciences, University of Waterloo, Ontario, Canada \\ ${ }^{\mathrm{d}}$ Department of Biological Sciences, Bauru School of Dentistry, University of São Paulo, USP, Bauru, Brazil
}

\section{A R T I C L E I N F O}

\section{Article history:}

Received 1 October 2015

Received in revised form 9 December 2015

Accepted 21 December 2015

Available online 28 December 2015

\section{Keywords:}

Skeletal muscle

Glucocorticoids

Flexor hallucis longus muscle

MuRF-1

\begin{abstract}
A B S T R A C T
This study was designed to describe the time-course changes of catabolic proteins following muscle atrophy induced by 10 days of dexamethasone (DEX). Rats underwent DEX treatment for 1, 3, 5, 7 and 10 days. Body weight (BW) and lean mass were obtained using a dual energy X-ray absorptiometry (DEXA) scan. Muscle ringer finger1 (MuRF-1), atrogin-1 and myostatin protein levels were analyzed in the tibialis anterior (TA), flexor hallucis longus (FHL) and soleus muscles. DEX treatment reduced lean mass since day- 3 and reduced BW since day-5. Specific muscle weight reductions were observed after day-10 in TA $(-23 \%)$ and after day-5 in FHL $(-16 \%,-17 \%$ and $-29 \%$, for days 5,7 and 10 , respectively). In TA, myostatin protein level was $36 \%$ higher on day- 5 and its values were normalized in comparison with controls on day-10. MuRF-1 protein level was increased in TA muscle from day-7 and in FHL muscle only on day-10. This study suggests that DEX-induced muscle atrophy is a dynamic process which involves important signaling factors over time. As demonstrated by DEXA scan, lean mass declines earlier than BW and this response may involve other catabolic proteins than myostatin and MuRF-1. Specifically for TA and FHL, it seems that myostatin may trigger the catabolic process, and MuRF-1 may contribute to maintain muscle atrophy. This information may support any intervention in order to attenuate the muscle atrophy during long period of treatment.
\end{abstract}

(c) 2015 Elsevier Inc. All rights reserved.

\section{Introduction}

Muscle wasting is a common side-effect after chronic treatment with corticosteroids or desnervation [1-4]. Muscle atrophy is normally maintained via a tight control of signaling processes involved in both synthesis and degradation of muscle proteins $[5,6]$, but the time-course of molecular signaling mechanisms involved in this response is not completely established.

Abbreviations: DEX, dexamethasone; C, control; CEUA, Committee for Ethical Use of Animals; DEXA, dual energy X-ray absorptiometry; MuRF-1, muscle ring finger1; Atrogin-1, muscle atrofic F-box; TA, tibialis anterior muscle; FHL, flexor hallucis longus muscle; SOL, soleus muscle; BW, body weight; IRS-1, insulin receptor substrate; GLUT-4, glucose transporter; AKT, serine/threonine protein kinase B; TGF- $\beta$, growth stimulating factor $\beta$; FOXO, Forkead transcription factors; PMSF, phenylmethylsulfonyl fluoride; IGF-1, insulin-like growth factor-1; mTOR, mammalian target of rapamycin.

* Corresponding author at: Department of Physical Education, Science Faculty, UNESP - Universidade Estadual Paulista, Bauru, SP, Brazil.

E-mail address: slamaral@fc.unesp.br (S.L. Amaral).
Previous studies have shown that myostatin, atrogin-1 and MuRF-1 could be involved in muscle atrophy induced by chronic treatment with DEX, however, it is unclear if these proteins respond equally to DEX treatment or if there is a time-dependent effect. Gilson et al. [7] have suggested that myostatin has a crucial role on DEX-induced muscle atrophy, since myostatin-knockout mice did not present muscle atrophy induced by 10 days of DEX treatment. However, Ma et al. [8] have previously demonstrated that myostatin mRNA and protein level were pronounced after 5 days of DEX treatment, but the overexpression was not sustained after 10 days.

Much attention has been given to MuRF-1 and atrogin- 1 since there is substantial evidence suggesting that muscle proteolysis may result from ubiquitin-proteasome system activation [1,6,9-11]. We have recently demonstrated that MuRF-1 but not atrogin-1 protein level is increased in skeletal muscle after 10 days of DEX treatment [4]. This result is supported by Baehr et al. [12] who have demonstrated an essential role of MuRF-1 in 
DEX-treated animals, since a preventive effect was observed on muscle atrophy after glucocorticoid treatment in MuRF-1 knockout mice. Other authors, on the other hand, have shown an increase in atrogin-1 mRNA after DEX treatment; however, these studies used high doses compared with ours. Currently, there is no consensus about the exact mechanism responsible for muscle atrophy induced by DEX treatment. It seems that some proteins may be responsible for triggering this effect whereas others may be responsible for the maintenance of muscle atrophy. Therefore, the aim of this study was to describe the time-course changes of catabolic protein following experimental muscle atrophy induced by 10 days of DEX treatment.

\section{Experimental}

\subsection{Animals}

Adult male Wistar rats (200-250 g) were obtained from Center for Research and Production Facilities of UNESP (Botucatu, SP, Brazil) and housed in group cages ( 5 in each) at the Animal Facility Maintenance from Faculty of Science, UNESP of Bauru. All rats were maintained under controlled environmental conditions $(12 \mathrm{~h}$ dark-light cycle, $22^{\circ} \mathrm{C}$ of temperature) with standard diet (Biobase, Brazil) and water ad libitum. All methods were approved by the Committee for Ethical Use of Animals (CEUA) of Universidade Estadual Paulista - UNESP, Araçatuba (approved protocol \#201202253).

Rats were divided into two groups: DEX, which received DEX treatment for $1,3,5,7$ or 10 days (DEX, Decadron ${ }^{\circledR}, 0.5 \mathrm{mg} / \mathrm{kg}$ of body weight, i.p, $n=40$ ) and control animals, who received saline during the same period ( $C, n=42)$. DEX and saline injections were daily given at the same time (7:00-8:00 h a.m.). Body weight was daily measured during DEX treatment.

\subsection{Dual energy X-ray absorptiometry (DEXA) method}

Measurements of body weight and lean mass of rats were obtained using a dual energy X-ray absorptiometry scan (DEXA, HOLOGIC, WI, USA), which is considered the standard clinical method to evaluate total body composition. Each anesthetized rat was positioned ventrally with arms separated from the trunk (Fig. 1, panel A) and the whole body was scanned by DEXA, using a specifically designed software version for small animals (Hologic Apex Software, version 3.2, USA).

\subsection{Blood glucose determination}

After an overnight fasting (12 h), blood samples were obtained from the tail puncture and the blood glucose was analyzed using glucose testing strips and a digital glucometer (One Touch Ultra - Johnsons \& Johnsons ${ }^{\circledR}$, USA).

\subsection{Tissue harvesting}

Tibialis anterior (TA), flexor hallucis longus (FHL) and soleus (SOL) muscles were removed and weighed just after euthanasia by an overdose of anesthesia (Ketamine $160 \mathrm{mg} / \mathrm{kg}$ and Xylasin $20 \mathrm{mg} / \mathrm{kg}$ ). These muscles were chosen since FHL is composed predominantly of myosin heavy chain $2 \mathrm{a} / \mathrm{x}$ (MHC IIa/x) with a small percentage of myosin heavy chain $2 \mathrm{~b}$ (MHC IIb) $[13,14]$, TA muscle is composed mainly of MHCIIb and soleus muscle is composed mainly of slow-fiber type I and IIa. Tibia bone length was measured for muscle weight normalization, since DEX treatment significantly reduces body weight. Muscles were immediately frozen and then stored at $-80^{\circ} \mathrm{C}$ for further analysis.

\subsection{Western blot procedures}

Samples of TA, FHL and SOL were homogenized in RIPA solution with $0.1 \%$ protease inhibitor cocktail and $1 \%$ of phenylmethylsulfonyl fluoride (PMSF), using a Polytron homogenizer as we previously published [4]. Samples were centrifuged at $10,000 \times g$ per $5 \mathrm{~min}$ and then the supernatant was collected and stored at $-20^{\circ} \mathrm{C}$ for future analysis. Bradford assays were used to determine the protein concentration (Bio-Rad Kit, Protein Assay Standart II, Hercules, CA, USA) as previously published [5-10]. Absorbance values were determined using a spectrophotometric plate reader (BMG labtech, Spectro Star Nano, Germany). Western blotting procedures were performed according to previously published studies $[3,4]$. In summary, $50-80 \mu \mathrm{g}$ of protein were separated using $10 \%$ denaturing polyacrylamide gels with running buffer for $60 \mathrm{~min}$. These gels were then transferred to nitrocellulose membranes using a transfer buffer for $90 \mathrm{~min}$. The equal protein loading was confirmed using a $0.5 \%$ Ponceau S staining solution. Membranes were blocked with bovine serum albumin (BSA, 1.5\%) in Tris-buffered saline with Tween (TBS-T) for 40 s. SNAP i.d. ${ }^{\circledR} 2.0$ Protein Detection system (Millipore) was used to incubate the membranes with primary and secondary antibodies for $10 \mathrm{~min}$ as previously published by Macedo et al. [4]: primary antibodies in 3\% BSA: atrogin-1 (Abcam \#a74023, 1:1000), MuRF-1 (Santa Cruz (C-20) sc27642, 1:1000) and myostatin/GDF-8 (Novus Biological (3E7) 1:500). Secondary antibodies were diluted in 1\% BSA: atrogin-1 (Jackson Immuno Research \#111-035-003, IgG anti-rabbit, 1:10,000), MuRF-1 (Jackson Immuno Research \#705-035-003, anti-donkey, 1:10,000) and myostatin (Jackson Immuno Research \#115-035-003, IgG anti-mouse, 1:5000) for $10 \mathrm{~min}$. The antibody was detected by enhanced chemiluminescence (Super signal West Pico, Pierce ${ }^{\circledR}$, Rockford, Illinois, USA) and the membranes were exposed to a radiographic film. The bands were analyzed using a computer program (Scion Image Corporation, version Beta 4.02, Frederick, Maryland, USA) and the values were expressed as percentage of control.

\subsection{Statistics}

The results are presented as mean \pm standard error of mean. ANOVA Two-way with repeated measures was used (considering treatment and days as factors). The association between body weight and lean mass/muscle and protein level were assessed using Pearson correlation coefficient. Tukey post hoc test was used when necessary $(p<0.05)$. Software used: SigmaStat v.3.1.

\section{Results}

Fig. 1 illustrates DEXA scans of 2 representative animals (panel A, days 0,5 and 10), lean mass (panel B) and body weight (BW, panel C) of rats through 10 days of DEX treatment, obtained by DEXA scan analysis. As shown in Fig. 1B, significant reductions in lean mass were observed since day $3(-12 \%,-22 \%,-23 \%$ and $-28 \%$, for days $3,5,7$ and 10 , respectively, compared with their respective controls). All rats started the experimental protocol with similar BW $(355 \pm 11 \mathrm{~g}$ vs $346 \pm 10 \mathrm{~g}$, for C vs DEX, respectively). DEX treatment resulted in BW reductions from day-5 $(-18 \%)$ up to days $7(-21 \%)$ and $10(-27 \%)$, when compared with respective control groups (Fig. 1, panel C), as suggested by DEXA images (panel A). We found a positive and highly significant correlation between lean mass and body weight of rats on days 5 $(r=0.9466)$ and $10(r=0.9898)$.

All groups presented similar values of fasting blood glucose before the experimental protocol. As shown in Table 1, DEX treatment significantly increased blood glucose since the first day of 
A

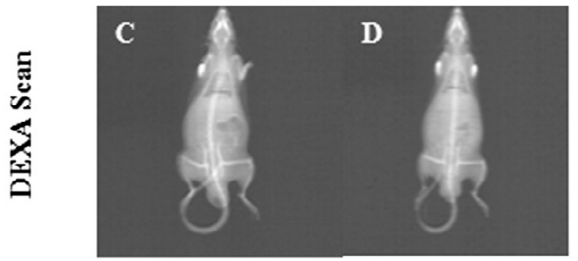

Before

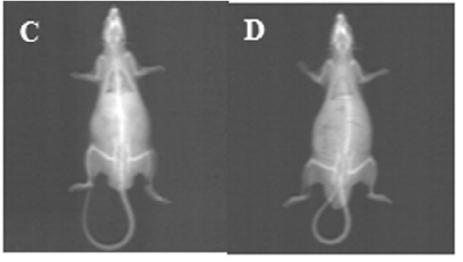

5 Days

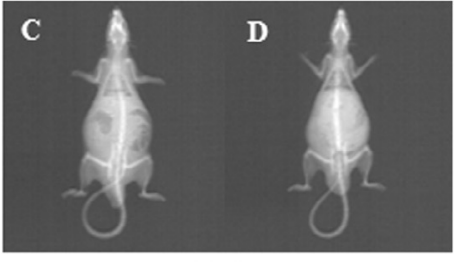

10 Days

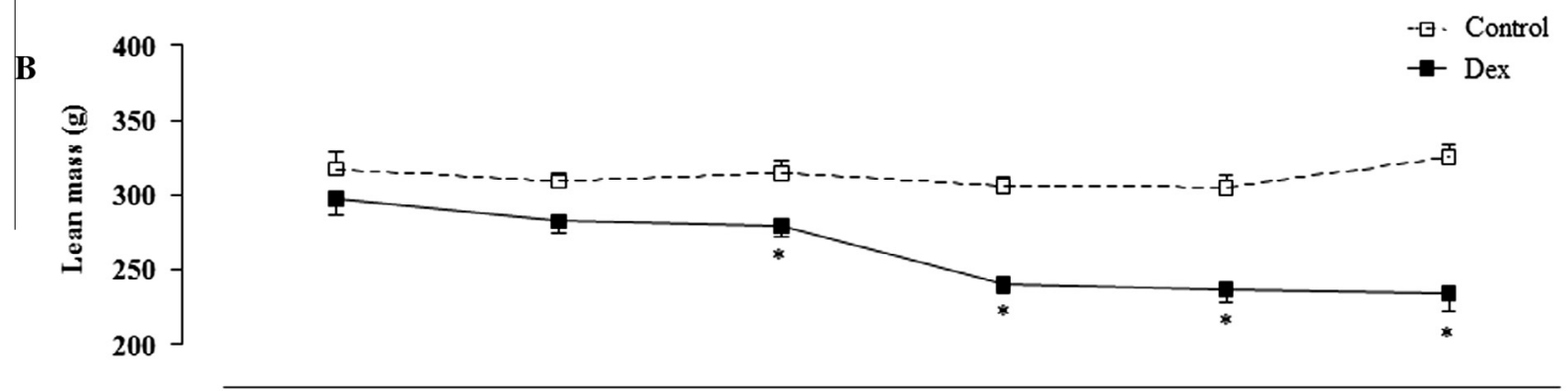

C

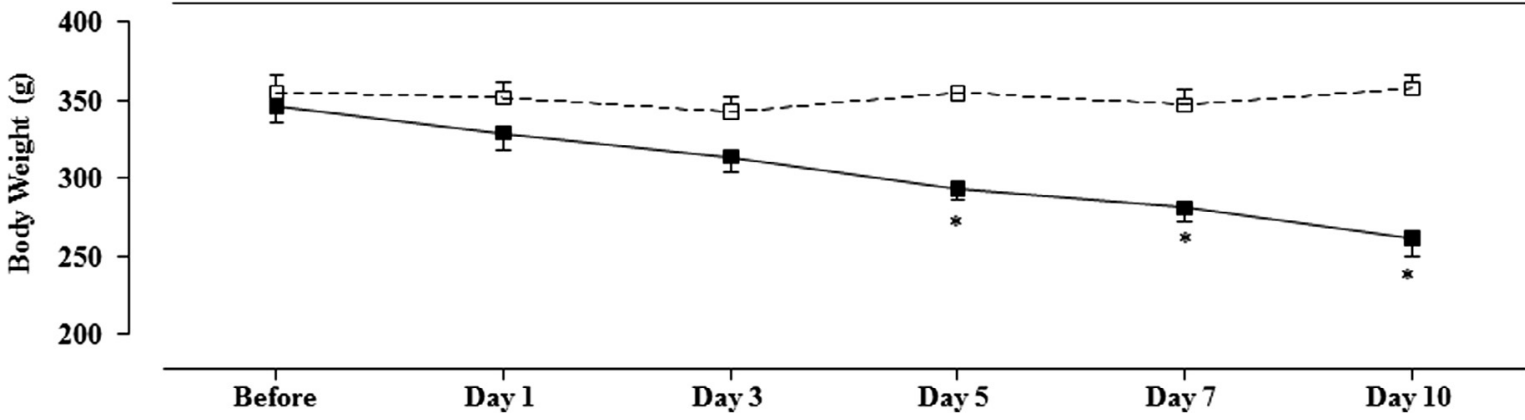

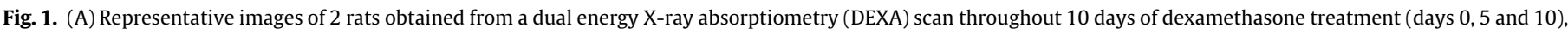

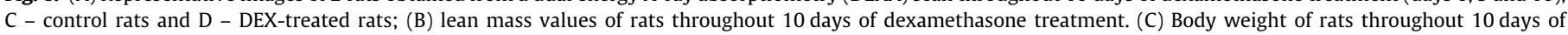
dexamethasone treatment; dexamethasone treatment (DEX, $n=5)$ and control group $(C, n=5)$. Significance: ${ }^{*} v s$ control, $p<0.05$.

Table 1

Fasting blood glucose during experimental protocol in all groups.

\begin{tabular}{ccccc}
\hline & Before & Day $1(\mathrm{mg} / \mathrm{dL})$ & Day $5(\mathrm{mg} / \mathrm{dL})$ & Day $10(\mathrm{mg} / \mathrm{dL})$ \\
\hline C & $76 \pm 2$ & $83 \pm 4$ & $77 \pm 2$ & $78 \pm 4$ \\
D & $78 \pm 4$ & $107 \pm 5^{*}$ & $133 \pm 13^{*}$ & $109 \pm 10^{*}$ \\
\hline
\end{tabular}

$\mathrm{C}=$ control $(n=7-10) ; \mathrm{D}=$ rats treated with dexamethasone $(n=7-9)$. Significance: ${ }^{*}$ vs control group, $p<0.05$.

treatment $(+22 \%,+43 \%,+28 \%$ for days 1 , 5and 10 , respectively, compared with their respective controls).

Figs. 2 and 3 illustrate skeletal muscle weight (A) and protein levels of myostatin (B), atrogin-1 (C) and MuRF-1 (D). Fig. 2A shows that DEX treatment promoted a significant TA muscle mass reduction on day 10 ( $-23 \%$ vs control group). Myostatin protein levels (Fig. 2B) was 37\% higher than control on day 5, but it returned to baseline levels on day 10. DEX treatment did not change atrogin-1 protein level, as shown in Fig. 2C; however, after 7 days of DEX treatment, an increase in MuRF-1 protein level $(+44 \%)$ was observed, which was kept elevated up to day 10 ( $+36 \%$ vs control, Fig. 2D).

Muscle mass of FHL was also reduced by DEX treatment since day-5 ( $-16 \%,-17 \%$ and $29 \%$, for days 5,7 and 10 , respectively, $v$ s control) as shown in Fig. 3A. On day-5, myostatin protein level in DEX group was 27\% higher than control, however, it did not reach significant level (Fig. 3B, $p<0.06$ ). Atrogin-1 protein level remained unchanged in FHL muscle during all 10 days of DEX treatment, as illustrated in Fig. 3C; however, DEX treatment induced a MuRF-1 protein level increase (44\%) only on day-10 compared with control (Fig. 3D). DEX treatment did not change SOL muscle weight. Accordingly, it did not change myostatin, atrogin-1 or MuRF-1 protein level.

Fig. 4 didactically illustrates the relationship between myostatin, atrogin- 1 and MuRF-1 protein level with the respective muscle weights (TA and FHL) on days 5 and 10 of DEX treatment. As shown, high myostatin protein level was associated with lower TA $(r=0.6088)$ and FHL $(r=0.5918)$ muscle weights only on day-5. On the other hand, high MuRF-1 protein level was associated with lower TA $(r=0.7574)$ and FHL $(r=0.7274)$ muscle weights on day10. Atrogin-1 was not associated with muscle weight at any moment of DEX treatment. Soleus muscle did not show any correlation between proteins level and muscle weight (myostatin and SOL/tibia: $r=0.0316$ and $r=0.3324$; atrogin and SOL/tibia: $r=0.2769$ and $r=0.0787$; MuRF-1 and SOL/tibia: $r=0.264$ and $r=0.0316$, for day 5 and day 10 , respectively).

\section{Discussion}

The main results of this work were that chronic DEX treatment caused body weight reduction which was associated with lower lean mass. The descriptive time-course of atrophic protein levels suggests that myostatin increases on day 5 and MuRF- 1 increases only after 10 days of DEX treatment.

Although DEX is widely used to treat allergies and inflammations, it alters carbohydrates, lipids and proteins metabolisms, triggering several side-effects such as peripheral insulin resistance, hypercholesterolemia, hypertension and muscle atrophy $[3,4,8,15,16]$. Hyperglycemia seems to be a common side effect of 

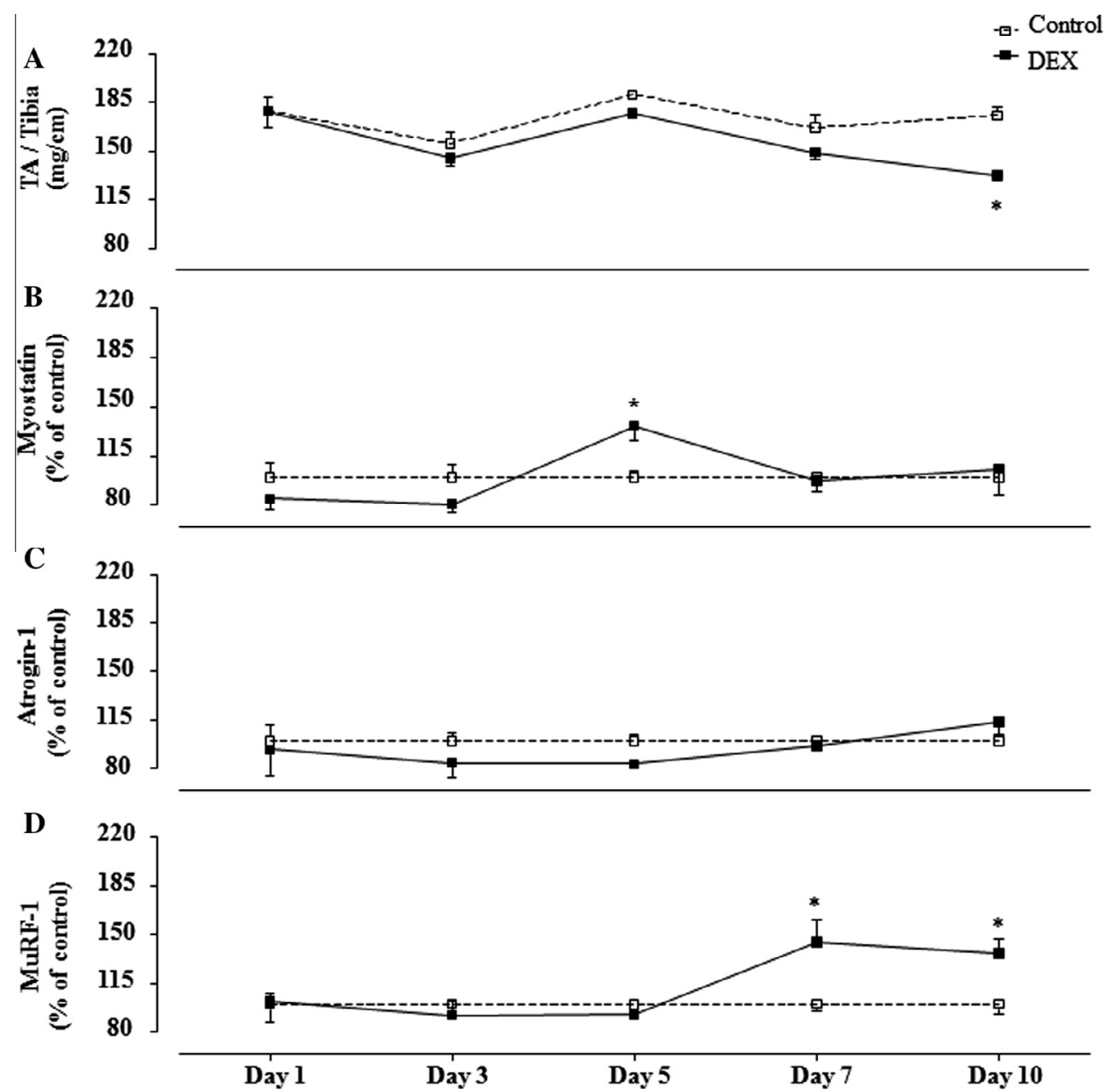

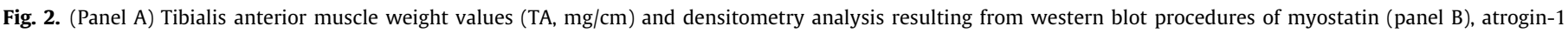

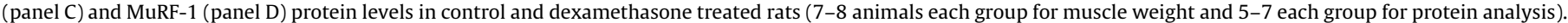
Significance: * $v$ s control, $p<0.05$.

DEX treatment and it can occur even after one single dose, as shown by the results of the present study and other authors $[17,18]$. This fast response is normally maintained during chronic treatments, as also observed by this present work and several authors $[3,4,16,18-20]$, which is mediated by reductions in important proteins involved in skeletal muscle glucose uptake such as IRS-1, AKT and GLUT-4.

In this study, we demonstrated, for the first time, a time-course of lean mass and BW profile throughout 10 days of DEX treatment, using the gold standard dual energy X-ray absorptiometry (DEXA) scan. As observed in the present study, lean mass (DEXA scan) was already lower after only 3 days of DEX treatment, while BW of DEX-treated rats was significantly lower than control animals after the day 5 of DEX treatment. Even though TA and FHL muscle mass was reduced only after 5-10 days, it is important to note that results from DEXA scan represent a sum of all muscles in the body and maybe different muscles respond at different times to DEX treatment. Since bone mass did not significantly change throughout this treatment (data not shown), it seems reasonable to think that muscle mass loss may occur before BW reduction. In agreement, Gilson et al. [7] have shown that when muscle atrophy is avoided, in myostatin-knockout mice, BW is not reduced in animals treated with DEX, and these authors suggest that muscle mass may influence BW loss in DEX treatment. In fact, our results showed a strong correlation between lean mass and body weight. In contrast to our results, Auclair et al. [21] and Cho et al. [9] have demonstrated that BW reduce much earlier than muscle mass, thus suggesting that there is a discordance between BW reduction and muscle atrophy.

Body weight reduction induced by chronic treatment with DEX is usually reported $[3,4,10,18,22]$. The mechanism responsible for this response is not completely understood, but it may be somewhat explained by food intake reductions $[4,7,8,23]$ since DEX inhibits the production of ghrelin and increases plasma levels of leptin $[24,25]$, which contribute to an increase in satiety signaling in the central nervous system. However, it seems that food intake reductions contribute to trigger BW reductions and other mechanisms are involved in the maintenance of this response, since we have shown that reduced food intake normalizes after 8 days of treatment $[4,26]$.

Although several authors have already demonstrated muscle atrophy induced by DEX (or other glucocorticoid), the mechanism responsible for this response is not completely understood. It may be mediated by increases in catabolic proteins $[1,4,9,26,27]$. It is important to note that there is a huge difference between DEX doses and treatment periods in the literature and most of them show a final end point only. Therefore, this study was performed in order to describe the time-course of skeletal muscle atrophic protein levels throughout a period of $1,3,5,7$ and 10 days of DEX treatment.

Ma et al. [8] first demonstrated that muscle weight loss induced by 5 days of DEX treatment was associated with an up-regulation 


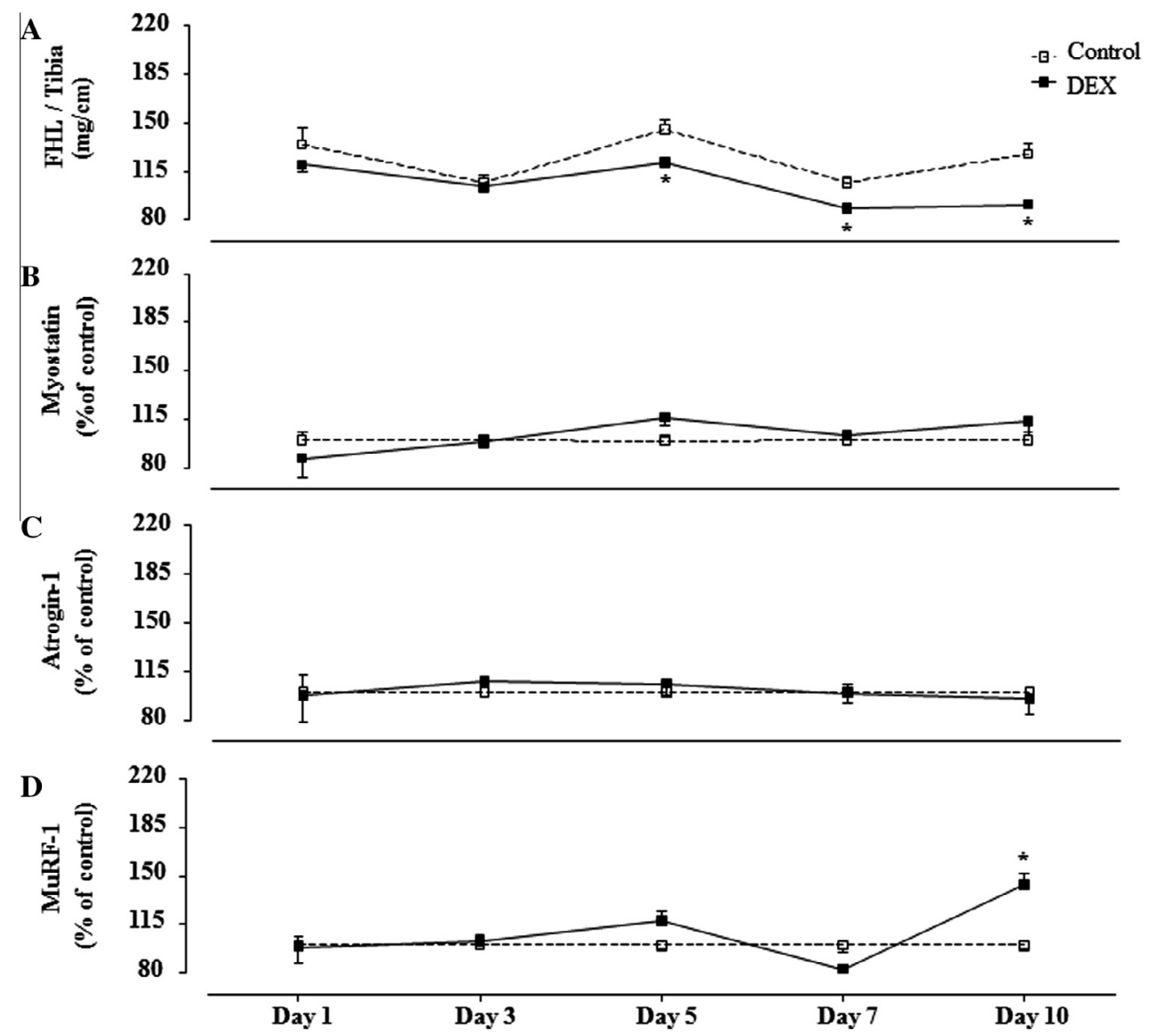

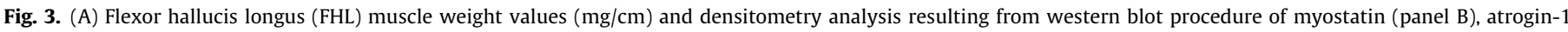

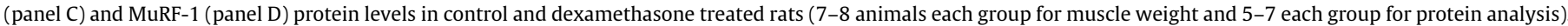
Significance: * vs control, $p<0.05$.
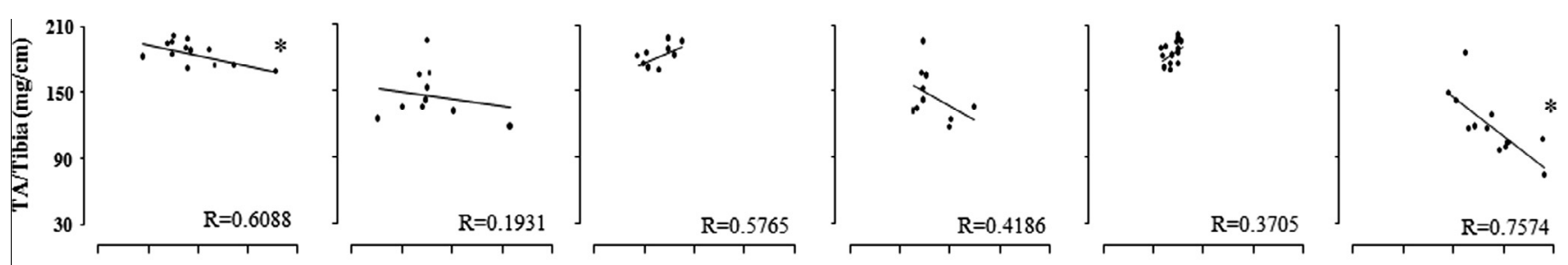

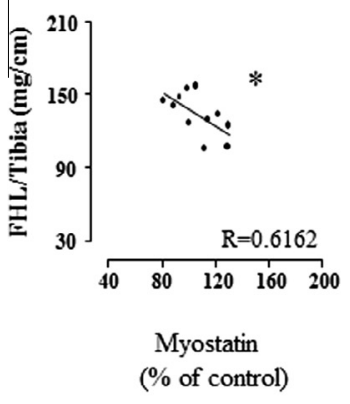

Day 5

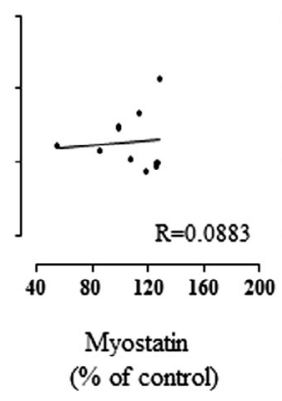

Day 10

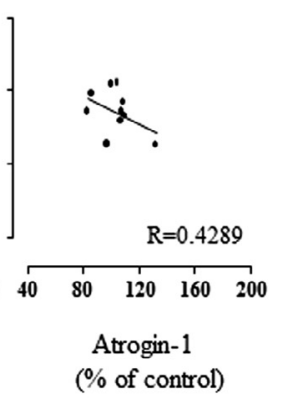

Day 5

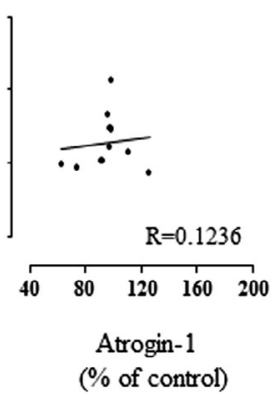

Day 10

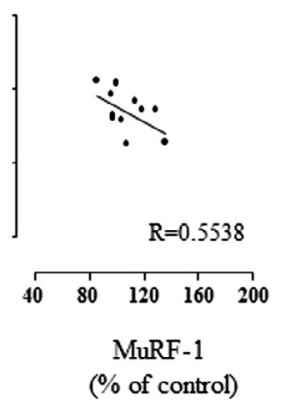

Day 5

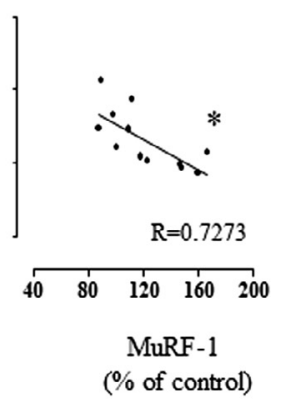

Day 10

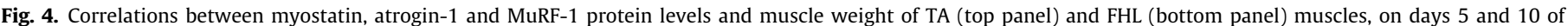
dexamethasone treatment $(p<0.05)$. Significance: "means significant correlation using Pearson correlation test, $p<0.05)$.

of myostatin mRNA in vivo. These authors have suggested that myostatin, which is involved in several conditions of skeletal muscle mass loss [28], could be one of the factors responsible for mus- cle weight loss observed in DEX-treated rats. Indeed, Gilson et al. [7] confirmed this hypothesis since the deletion of myostatin gene in mice prevented the reduction of muscle mass and also the BW 
loss induced by DEX treatment. In accordance, the results of the present study confirm that skeletal muscle myostatin protein level increased after 5 days of DEX treatment (at least on TA muscle, since the $16 \%$ increase of myostatin protein level in FHL muscle was not significant). Myostatin is a TGF- $\beta$ family member which is highly expressed by skeletal muscle [14]. It has been suggested that myostatin causes muscle atrophy through inhibition of AKT pathway by activation of smad 2/3 [29-31]. In accordance, our previous studies have shown lower AKT protein level in atrophic TA muscle after DEX treatment $[4,16]$. It is important to note that lean mass (DEXA scan) decreases even earlier than myostatin increases, which could indicate that maybe other proteins, different from those analyzed in this present work, may also be involved in the fast reduction of lean mass.

However, it seems that other mechanisms are responsible for the maintenance of muscle atrophy, since after 10 days of DEX treatment, myostatin protein level normalized, as already shown by Ma et al. [8].

Even though myostatin has been implicated in muscle atrophy, several authors $[1,9,27,32]$ have shown that muscle atrophy induced by DEX could be mediated by gene and protein increases of two important E3 ligases, namely atrogin-1 and MuRF-1. Recently, we demonstrated that muscle atrophy induced by DEX may be mediated by decreases in AKT protein level associated with increases in MuRF-1 and maintenance of atrogin- 1 protein level [4].

In the present work, in agreement with our previous results, atrogin-1 protein level remained unchanged over the 10 days of treatment with DEX. In contrast, Cho et al. [9], Baehr et al. [12] and Watson et al. [10] have shown that glucocorticoid increases atrogin-1 expression in TA muscle associated with atrophic response in muscles. However, in these studies, the dose (higher than in the present work) and the route of administration were different than in the present work, which may explain the different results.

Protein abundance of the other muscle-specific ubiquitin E3 ligase, MuRF-1, increased in a different time as compared with myostatin in the present work (TA and FHL), which agrees with Sartori et al. [29], who proposed that TGF- $\beta /$ smad 2 pathway (myostatin pathway) induced muscle atrophy independent of MuRF-1 activation. Similar increments of MuRF-1 after DEX treatment were observed in gastrocnemius [1], plantaris [23] and TA [10,12] muscles. We can speculate that this late activation could be a consequence of an inhibition of IGF-1/AKT pathway, induced earlier by myostatin [30], and that dephosphorylated Akt could result in the activation of existing FOXO transcription factors to induce MuRF-1 expression [6,9,29]. Taken together these results can suggest that this model of muscle atrophy induced by DEX was associated mainly with increments of myostatin (5 days) and MuRF-1 (10 days), with no participation of atrogin-1, which partially agrees with Baehr et al. [12] who demonstrated that MuRF-1 has a crucial role in muscle atrophy induced by DEX instead of atrogin-1.

Soleus muscle remained unchanged throughout 10 days of DEX treatment, which is in agreement with previous publications showing that DEX or any glucocorticoids have different catabolic effects among slow fiber-type muscle (like soleus), and fast fibertype (like TA or FHL muscles) [2,21,33].

This time-course approach allows us to suggest that BW decline may be influenced by a dynamic process of protein catabolism. It seems that protein degradation starts quite fast, as already demonstrated by Auclair et al. [21], which could determine the decrease in lean mass, observed on day 3 by DEXA scan. In addition, the association graphs presented in this time-course study contribute to the understanding that myostatin pathway (myostatin type II receptor/smad2/AKT pathway) would be associated with the earlier stimulus (day 5) to the protein degradation process in TA and FHL muscles. The same figure also suggest that activation of MuRF-1, induced by FOXO pathway (dephosphorylated AKT) would facilitate the maintenance on muscle atrophy induced by chronic treatment with DEX, as observed on day 10 .

In conclusion, this time-course study suggests that DEXinduced muscle atrophy is a dynamic process which involves important signaling factors over time. As demonstrated by DEXA scan, lean mass declines earlier than BW and this response may involve other catabolic proteins than Myostatin and MuRF-1. Specifically for TA and FHL, it seems that myostatin may trigger the catabolic process, and MuRF-1 may contribute to maintain muscle atrophy. This information may support any intervention in order to attenuate the muscle atrophy during long period of treatment.

\section{Acknowledgments}

This study was supported by São Paulo Research Foudation (FAPESP \#2011/21522-0 grant to S.L.A.). A.G.M. was a recipient of a scholarship from the National Council for Scientific and Technological Development (CNPq, process \#130232/2011-4). A.L.O.K. was a recipient of a scholarship from the São Paulo Research Foundation (FAPESP \#2012/21820-3). L.M.S. was a recipient of a scholarship from São Paulo Research Foundation (FAPESP \#2012/038169 ). The authors thank the technical support from Bruno A. Viscelli for his help with DEXA scan measurements.

\section{References}

[1] S.C. Bodine, E. Latres, S. Baumhueter, V.K. Lai, L. Nunez, B.A. Clarke, et al., Identification of ubiquitin ligases required for skeletal muscle atrophy, Science 294 (2001) 1704-1705

[2] A.M. Ahtikoski, E.M. Riso, S.O. Koskinen, J. Risteli, Regulation of type IV collagen gene expression and degradation in fast and muscle during dexamethasone treatment and exercise, Pflugers Arch. 448 (1) (2003) 123-130.

[3] M. Barel, O.A. Perez, V.A. Giozzet, A. Rafacho, J.R. Bosqueiro, S.L. do Amaral, Exercise training prevents hyperinsulinemia, muscular glycogen and muscle atrophy induced by dexamethasone treatment, Eur. J. Appl. Physiol. 108 (2010) 999-1007.

[4] A.G. Macedo, A.L.O. Krug, N.A. Herrera, A.S. Zago, J.W.E. Rush, S.L. Amaral, Low intensity resistance training attenuates dexamethasone induced atrophy in the flexor hallucis longus muscle, J. Steroid Biochem. Mol. Biol. 143 (2014) 357364.

[5] O. Schakman, H. Gilson, J.P. Thissen, Mechanisms of glucocorticoid-induced myopathy, J. Endocrinol. 197 (1) (2008) 1-10.

[6] M.A. Egerman, D.J. Glass, Signaling pathways controlling skeletal muscle mass, Crit. Rev. Biochem. Mol. Biol. 49 (1) (2014) 59-68.

[7] H. Gilson, O. Schakman, L. Combaret, P. Lause, L. Grobet, D. Attaix, Myostatin gene deletion prevents glucocorticoid-induced muscle atrophy, Endocrinology 148 (2007) 452-460.

[8] K. Ma, C. Mallidis, S. Bhasin, V. Mahabadi, J. Artaza, N. Gonzalez-Cadavid, et al., Glucocorticoid-induced skeletal muscle atrophy is associated with upregulation of myostatin gene expression, Am. J. Physiol. Endocrinol. Metab. 285 (2003) 363-371.

[9] J.E. Cho, M. Fournier, X. Da, M.I. Lewis, Time course expression Foxo transcription factors in skeletal muscle following corticosteroid administration, J. Appl. Physiol. 108 (2010) 137-145.

[10] M.L. Watson, L.M. Baehr, H.M. Reichardt, J.P. Tuckermann, S.C. Bodine, J.D. Furlow, A cell-autonomous role for the glucocorticoid receptor in skeletal muscle atrophy induced by systemic glucocorticoid exposure, Am. J. Physiol. Endocrinol. Metab. 302 (2012) 1210-1220.

[11] A.J. Murton, D. Constantin, P.L. Greenhaff, The involvement of the ubiquitin proteasome system in human skeletal muscle remodelling and atrophy, Biochim. Biophys. Acta 1782 (12) (2008) 730-743.

[12] L.M. Baehr, J.D. Furlow, S.C. Bodine, Muscle sparing in muscle RING finger 1 null mice: response to synthetic glucocorticoids, J. Physiol. 19 (2011) 47597761.

[13] T.A. Hornberger, R.P. Farrar, Physiological hypertrophy of the FHL muscle following 8 weeks of progressive resistance exercise in the rat, Can. J. Appl. Physiol. 29 (1) (2004) 16-31.

[14] S.J. Lee, Regulation of muscle mass by myostatin, Annu. Rev. Cell Dev. Biol. 20 (2004) 61-86.

[15] A. Rafacho, V.A. Giozzet, A.C. Boschero, J.R. Bosqueiro, Functional alterations in endocrine pancreas of rats with different degrees of dexamethasone-induced insulin resistance, Pancreas 36 (3) (2008) 284-293. 
[16] T.J. Dionísio, J.C. Louzada, B.A. Viscelli, E.J. Dionísio, M.A. Barel, A.O. Perez, et al., Aerobic training prevents dexamethasone-induced peripheral insulin resistance, Horm. Metab. Res. 46 (7) (2014) 484-489.

[17] D. Qi, T. Pulinilkunnil, D. An, S. Ghosh, A. Abrahani, J.A. Pospisilik, et al., Single dose dexamethasone induces whole-body insulin resistance and alters both cardiac fatty acid and carbohydrate metabolism, Diabetes 53 (2004) 17901797.

[18] G. Kewalramani, P. Puthanveetil, M.S. Kim, F. Wang, V. Lee, N. Hau, et al., Acute dexamethasone-induced increase in cardiac lipoprotein lipase requires activation of both Akt and stress kinases, Am. J. Physiol. Endocrinol. Metab. 295 (1) (2008) E137-E147.

[19] C.H. Pinheiro, W.M. Sousa Filho, J.d. Oliveira Neto, J Marinho Mde, R. Motta Neto, M.M. Smith, Exercise prevents cardiometabolic alterations induced by chronic use of glucocorticoids, Arq. Bras. Cardiol. 93 (4) (2009) 400-408.

[20] J. Ruzzin, A.S. Wagman, J. Jensen, Glucocorticoid-induced insulin resistance in skeletal muscles: defects in insulin signalling and the effects of a selective glycogen synthase kinase-3 inhibitor, Diabetologia 48 (10) (2005) 2119-2130.

[21] D. Auclair, D.R. Garrel, A. ChaoukiZerouala, L.H. Ferland, Activation of the ubiquitin pathway in rat skeletal muscle by catabolic doses of glucocorticoids, Am. J. Physiol. 272 (1997) 1007-1016.

[22] H. Nicastro, N.E. Zanchi, C.R. da Luz, W.M. de Moraes, P. Ramona, M.A. de Siqueira Filho, et al., Effects of leucine supplementation and resistance exercise on dexamethasone-induced muscle atrophy and insulin resistance in rats, Nutrition (2012) 1-7.

[23] H. Nicastro, B. Gualano, W.M. de Moraes, Painelli V. de Salles, C.R. da Luz, Costa A. dos Santos, et al., Effects of creatine supplementation on muscle wasting and glucose homeostasis in rats treated with dexamethasone, Amino Acids 42 (2012) 1695-1701.

[24] G. Tulipano, J.E. Taylor, H.A. Halem, R. Datta, J.Z. Dong, M.D. Culler, et al., Glucocorticoid inhibition of growth in rats: partial reversal with the fulllength ghrelin analog BIM-28125, Pituitary 10 (3) (2007) 267-274.
[25] J.W. Jahng, N.Y. Kim, V. Ryu, S.B. Yoo, B.T. Kim, D.W. Kang, et al., Dexamethasone reduces food intake, weight gain and the hypothalamic 5HT concentration and increases plasma leptin in rats, Eur. J. Pharmacol. 508 (2008) 64-70.

[26] A.L. Krug, A.G. Macedo, A.S. Zago, J.W. Rush, C.F. Santos, S.L. Amaral, High intensity training attenuates dexamethasone induced muscle atrophy, Muscle Nerve (2015), http://dx.doi.org/10.1002/mus.24906 (in press).

[27] D.T. Hwee, A.V. Gomes, S.C. Bodine, Cardiac proteasome activity in muscle ring finger-1 null mice at rest and following synthetic glucocorticoid treatment, Am. J. Physiol. Endocrinol. Metab. 301 (5) (2011) E967-E977.

[28] N.E. Brooks, K.H. Myburgh, K.B. Storey, Myostatin levels in skeletal muscle of hibernating ground squirrels, J. Exp. Biol. 214 (2011) 2522-2527.

[29] R. Sartori, G. Milan, M. Patron, C. Mammucari, B. Blaauw, R. Abraham, et al. Smad2 and 3 transcription factors control muscle mass in adulthood, Am. J. Physiol. Cell Physiol. 296 (2009) C1248-C1257.

[30] K. Hitachi, M. Nakatani, K. Tsuchida, Myostatin signaling regulates Akt acidity via the regulation of miR-486 expression, Int. J. Biochem. Cell Biol. 47 (2014) 93-103.

[31] J. Rodriguez, B. Vernus, I. Chelh, I. Cassar-Malek, J.C. Gabillard, A. HadjSassi, et al., A myostatin and the skeletal muscle atrophy and hypertrophy signaling pathways, Cell. Mol. Life Sci. 71 (22) (2014) 4361-4371.

[32] E. Castillero, N. Alamdari, S.H. Lecker, P.O. Hasselgren, Suppression of atrogin-1 and MuRF-1 prevented dexamethasone-induced atrophy of cultured myotubes, Metabolism 62 (10) (2013) 1495-1502.

[33] M.T. Falduto, S.M. Czerwinski, R.C. Hickson, Glucocorticoid-induced muscle atrophy by exercise in fast-twitch fibres, J. Appl. Physiol. 69 (3) (1990) 1058 1062. 